\title{
p-IVG: Probabilistic Inter-Vehicle Geocast for Dense Vehicular Networks
}

\author{
Khaled Ibrahim, Michele C. Weigle and Mahmoud Abuelela \\ Department of Computer Science \\ Old Dominion University \\ Norfolk, VA 23529-0162 \\ $\{$ ibrah_k, mweigle, eabu\}@cs.odu.edu
}

\begin{abstract}
In this paper, we study the effect of dense vehicular networks on data dissemination. When using intelligent broadcasting techniques, such as Inter-Vehicle Geocast, we have discovered the spatial broadcast storm problem in which multiple vehicles will be chosen to re-broadcast frames at nearly the same time, resulting in channel contention and collisions. We present a probabilistic version of IVG ( $p$-IVG) to address this problem. In $p$-IVG, which vehicles re-broadcast frames is probabilistic depending upon the traffic density surrounding the vehicles. We show that $p$-IVG solves the spatial broadcast storm problem and that using $p$-IVG results in improved reception rates, lower channel contention, and most importantly, faster dissemination of data to distant vehicles than IVG.
\end{abstract}

\section{INTRODUCTION}

Efficient data dissemination is considered one of the most important problems in VANETs. The standard method of data communication is broadcast. Each vehicle sends a frame and all vehicles within transmission range can receive and process the data. To spread data farther than the original sender's transmission range, flooding is the typical method of dissemination. Basic flooding is defined as the following, whenever a node receives a frame, it will re-broadcast it, resulting in redundant re-broadcasts because the neighbors of the re-broadcasting node may have already received the original frame. Moreover, when multiple nodes in the same vicinity receive a frame, they will all re-broadcast it, causing severe contention on the channel. In addition to the previous two problems, collisions are highly probable due to the lack of CTS/RTS and collision detection. So, blindly flooding will waste bandwidth by sending redundant frames that will probably collide. These three problems are collectively known as the broadcast storm problem [1].

In VANETs, the broadcast storm problem occurs only in case of dense networks and recently, many protocols [2]-[5] have been proposed to alleviate this problem. Most of these protocols are based on selecting the farthest vehicle(s) within transmission range of the original sender to be the re-broadcaster(s). The selection is achieved by assigning increasing re-broadcast probabilities or decreasing waiting times as the distance from the sending vehicle increases. We show an example in Figure 1. Based on these criteria, all the vehicles in the highlighted area will have almost the same re-broadcast probability or waiting time, so they will re-broadcast the frame at the same time. This will cause a broadcast storm locally in the highlighted area. Although, the generated storm is local, it will affect the

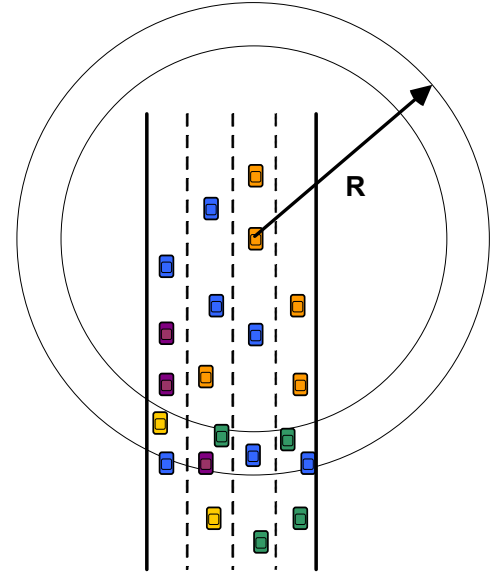

Fig. 1. Multiple vehicles at the boundary can cause a spatial broadcast storm

overall system performance. We term this the spatial broadcast storm problem.

In this paper, we study the impact of using Inter-Vehicle Geocast (IVG) [4] in case of dense VANETs and find that it will result in a spatial broadcast storm as shown in Figure 1. This work is the first to illustrate the existence of the spatial broadcast storm problem. To alleviate this problem we propose and develop an extension to IVG, probabilistic $I V G(p-I V G)$, to make the re-broadcast decision probabilistic based on awareness of the local topology, i.e., knowledge of surrounding vehicle density, instead of being deterministic. The local topology awareness is acquired through a light-weight topology sensing utility. We show that using $p$-IVG results in improved reception rates, lower channel contention, and most importantly, faster dissemination to distant vehicles.

\section{RELATED WORK}

Various solutions have proposed to handle the broadcast storm problem in mobile ad-hoc networks (MANETs) [1], [6], [7]. As VANETs have some very different characteristics from MANETs, namely higher mobility speeds and restricted network topologies and vehicle movement, the proposed solutions for the broadcast storm problem in MANETs are not applicable for VANETs.

The approaches that have been proposed to alleviate the broadcast storm problem in dense VANETs can be classified as 
probability-based, timer-based and priority-based approaches.

The main idea behind the probability-based techniques is that whenever a vehicle receives a frame, it will re-broadcast it after holding it for a certain waiting time, according to a probability $p$ that depends on how far this vehicle is from the sending vehicle. Distant vehicles will have higher probability $p$ to re-broadcast the received frames than nearby vehicles. This technique is called weighted $p$-persistence broadcasting and has been proposed by Wisitpongphan et al. [2].

There are two versions of the timer-based techniques: slotted time and continuous time. Wisitpongphan et al. [2] proposed the slotted 1-persistence broadcasting technique, dividing the waiting time into slots. When each vehicle receives a frame, it is assigned a time slot during which it should re-broadcast the frame with probability 1 if no one else had re-broadcasted it. As the distance increases, the receiving vehicle is assigned a shorter re-broadcast time slot. Also, the same authors proposed a slotted p-persistence broadcasting technique, in which during the assigned time slot the vehicle will re-broadcast the frame with a pre-determined probability $p$.

Briesemeister et al. [8] proposed a continuous time version of the timer-based technique, called role-based multicast. In this technique, whenever a vehicle receives a frame, it waits for a certain amount of time before re-broadcasting it. The longer the distance from the sender, the shorter the waiting time. This technique was only concerned with the sparsely connected networks to maximize the message reachability and does not handle the broadcast storm problem. Bachir et al. [4] proposed Inter-Vehicle Geocast (IVG), based on the same idea presented by Briesemeister et al. [8], but it handles the dense network situation.

In general, timer-based techniques have shown superiority over the probability-based techniques in mitigating the broadcast storm problem. In dense VANETs, the timer-based techniques re-broadcast the frame sooner (early re-broadcast) because the re-broadcast waiting time lessens with increasing distance from the sending vehicle, while the waiting time is constant in the probability-based techniques. Also, having an early re-broadcast will reduce the channel contention and redundant re-broadcasts because whoever hears the re-broadcast will cease its own re-broadcast. Within the timer-based techniques, the time continuous version known as IVG, is better than the slotted time techniques, because the slotted versions restrict the re-broadcast to be initiated only at certain times, which increases channel contention. Although IVG mitigates the broadcast storm problem, it suffers from the spatial broadcast storm problem, as it does not utilize the network topology information in the re-broadcast decision.

Torrent-Moreno et al. [3] proposed a priority-based broadcast scheme in which nodes that have a time-critical message to send will be assigned a higher priority to access the channel. In general, the priority-based techniques categorizes the network nodes into multiple classes with different priorities and schedules frame transmission accordingly. This technique reduces the contention on the channel access by allowing the higher priority nodes to access the channel before the other nodes, but it does not solve the broadcast storm problem.

Tonguz et al. [5] provided a comprehensive framework (DVCAST) to handle broadcasting in VANETs considering three possible traffic densities (dense, regular, and sparse). In dense traffic, they suggest using one of the timer-based techniques [2], while in sparse traffic they suggest using role-based multicast [8]. They did not suggest a specific technique to be used in case of regular density traffic since they define regular density as a mix of some vehicles sensing dense traffic and some vehicles sensing sparse traffic. So, each vehicle will use the technique appropriate to what it has sensed. Even though DVCAST appears to be a complete solution, it is still vulnerable to the spatial broadcast storm problem.

\section{Probabilistic Inter-Vehicle Geocast}

In this paper, we present probabilistic-IVG ( $p$-IVG), a lightweight extension to Inter-Vehicle Geocast (IVG) [4] for broadcasting messages in dense VANETs. The probability function in $p$-IVG is based on the density of surrounding vehicles. $p$-IVG adapts itself according to the current traffic conditions, in order to minimize the number of vehicles that will re-broadcast the received frame and, at the same time, minimize the probability of failing to re-broadcast the received frame. The surrounding vehicle density is detected through a light-weight local topology sensing utility.

In IVG, each vehicle starts a timer for each frame it receives. If the timer expires and the frame associated with this timer has not been re-broadcast by any other vehicle within transmission range, the vehicle re-broadcasts the frame. The timer value $T_{x}$ for vehicle $x$ is

$$
T_{x}=T_{\max } \cdot \frac{\left(R^{\varepsilon}-D_{s x}^{\varepsilon}\right)}{R^{\varepsilon}},
$$

where $R$ is the transmission range and $D_{s x}$ is the distance between vehicle $x$ and vehicle $s$, the sender of the frame. The authors of IVG suggest using $\varepsilon=2$ to generate a uniform timer value between $\left[0, T_{\max }\right]$, where $T_{\max }=200 \mathrm{~ms}$ [4].

By using IVG with its original settings, nodes that are close to each other at the boundary will have very similar, if not equal, timer values. This means that the nodes' timers will expire at almost the same time, and the nodes will re-broadcast the frame essentially simultaneously, resulting in collisions and thus, a spatial broadcast storm (Figure 1). The impact of using IVG on the system performance is presented in Section IV.

To alleviate this problem, we modified IVG by making the re-broadcasting of frames probabilistic based on the density of surrounding vehicles. In our probabilistic-IVG ( $p$-IVG), when a vehicle receives a frame, it first selects a random number in $[0,1]$. If the selected number is less than $\frac{1}{\text { density }}$, the timer is started. If not, then the frame will not be re-broadcasted by this vehicle. As the density increases, the number of nodes that will start their timers decreases.

\section{A. Effect of $\varepsilon$}

$\varepsilon$ is very critical parameter in the timer value equation of IVG. The authors of IVG provided no evaluation of how it 


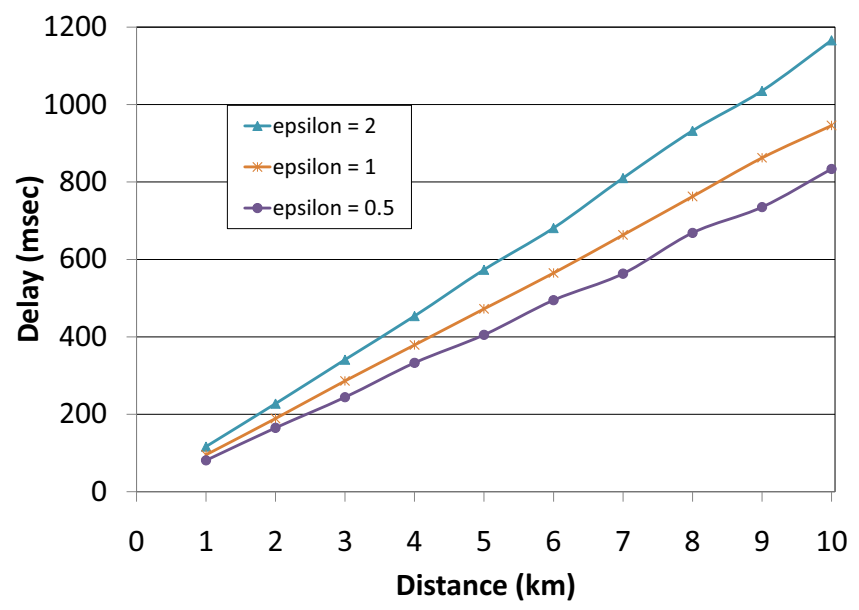

Fig. 2. The effect of $\varepsilon$ on frame propagation delay using $p$-IVG

affects the distribution of timer values. Here, we study this parameter carefully and investigate its effect on the whole system. Then for $p$-IVG, we tune this parameter in order to achieve maximum performance.

One of the main goals of the timer value equation is to reduce the waiting time, $T_{x}$, before frames are re-broadcast, and thus, the overall delay to send the frame to distant areas. In order to achieve this, smaller timer values should be generated as the distance from the original frame sender $\left(D_{s x}\right)$ increases.

Changing $\varepsilon$ has a great impact on the generated timer values. $\varepsilon>1$ generates a family of convex curves for the timer values, $\varepsilon<1$ generates a family for concave curves, and $\varepsilon=1$ generates a linear curve. Generally, the convex curve produces larger and sparser values than the concave curve, resulting in an increased time for frames to travel longer distances. Figure 2 shows how changing $\varepsilon$ affects the frame propagation delay for $p$-IVG. As $\varepsilon$ decreases, the propagation delay also decreases. But, through experimentation we found that if $\varepsilon<0.5$, although the frame propagation delay continued to decrease, contention increased. This was revealed through decreasing reception rates and increasing probabilities of backoffs when attempting to send frames. So, for the remainder of the paper, we use $\varepsilon=0.5$ and $T_{\max }=200 \mathrm{~ms}$.

\section{B. Local Topology Sensing}

The local network topology, or the density of vehicles within the transmission range, is sensed in $p$-IVG using a beacon broadcasting utility running in each vehicle. This utility broadcasts a beacon containing the vehicle's information (location, speed, etc.) twice a second. Each vehicle maintains a database to store the information of vehicles within its transmission range. Upon receiving a beacon, each vehicle updates its database with the new vehicle information. The database is checked every second. If there has been no update beacon received regarding a specific vehicle, this vehicle's record will be marked and its location information will be estimated using the most recent location and speed information. If the estimated location lies outside the transmission range, the vehicle's record will be purged from the database. If there has been no update beacon received regarding a specific vehicle for two consecutive checks (i.e., no update beacon received from the vehicle for two seconds), its record will be purged from the database. As these beacons are used for local topology sensing only, they are not re-broadcast. We do not consider these beacons as overhead because most vehicular applications already require each vehicle to broadcast its information frequently. These application messages, then, could be used to determine vehicle density.

\section{Vehicular Density}

Vehicular density is a key parameter that has a great influence on broadcasting techniques. Some of these techniques may fail in certain vehicular densities, so it is important to study the impact of density on our proposed technique, $p$-IVG. In case of IVG, as the density increases, the number of candidates for re-broadcasting a frame increases. This increases collisions, resulting in reduced reception rates. For $p$-IVG, the number of candidates for re-broadcasting a frame remain almost the same regardless of the density. This is because the number of candidates depends on both the density and the probability function that is inversely proportional to the density. So, as the density increases, the probability function decreases, and vice versa, keeping the number of candidates almost constant. This makes $p$-IVG produce stable performance regardless of the surrounding vehicular density.

\section{Evaluation}

\section{A. Simulation Setup}

We evaluate $p$-IVG using ASH (Application-aware SWANS with Highway mobility) [9], which is an extension of the SWANS (Scalable Wireless Ad hoc Network Simulation) vehicular network simulator [10], [11]. SWANS has been shown to be scalable and efficient, supporting large numbers of mobile nodes [12]. SWANS fully implements the IEEE 802.11a protocol, which we use as an approximation to IEEE 802.11p. Moreover, to produce realistic simulations, the ASH extensions to SWANS include implementations of the IDM (Intelligent Driver Model) vehicular mobility model [13] and the MOBIL (Minimizing Overall Braking decelerations Induced by Lane changes) lane changing model [14].

Table I summarizes our simulation settings. All vehicles in our simulations have a transmission range of $300 \mathrm{~m}$ [15], which is an acceptable standard range for DSRC. The roadway used is a four-lane unidirectional highway of length $100 \mathrm{~km}$. Vehicles enter the highway according to a Poisson distribution and travel at a maximum speed of $30 \mathrm{~m} / \mathrm{s}$. The simulation is run for 360 seconds, resulting in a total of 500 vehicles generated. In the 360 -second simulation runtime, the maximum distance traveled by any vehicle is $10 \mathrm{~km}$.

We evaluate $p$-IVG with three different traffic density scenarios. High density traffic has an average of 90 vehicles $/ \mathrm{km}$, medium density has an average of 66 vehicles $/ \mathrm{km}$, and low density has an average of 53 vehicles $/ \mathrm{km}$. These densities were gathered from the speed and traffic volume analysis 


\begin{tabular}{|l|l|}
\hline transmission range & $300 \mathrm{~m}$ \\
\hline highway length & $100 \mathrm{~km}$ \\
\hline max distance traveled & $10 \mathrm{~km}$ \\
\hline vehicles generated & 500 \\
\hline max speed & $30 \mathrm{~m} / \mathrm{s}$ \\
\hline simulation runtime & 360 seconds \\
\hline \hline high density & 90 vehicles $/ \mathrm{km}$ \\
\hline medium density & 66 vehicles $/ \mathrm{km}$ \\
\hline low density & 53 vehicles $/ \mathrm{km}$ \\
\hline
\end{tabular}

TABLE I

Simulation SETTINGS

\begin{tabular}{|c|c|c|c|}
\hline & \multicolumn{3}{|c|}{ Backoff Percentage } \\
\hline & Low Density & Medium Density & High Density \\
\hline$p$-IVG & $7.05 \%$ & $4.32 \%$ & $5.94 \%$ \\
\hline IVG & $31.09 \%$ & $35.94 \%$ & $42.67 \%$ \\
\hline Flooding & $80.83 \%$ & $84.38 \%$ & $92.11 \%$ \\
\hline
\end{tabular}

TABLE II

BACKOFF PERCENTAGE

performed by Wisitpongphan et al. [16] for the data collected by the Berkeley Highway Lab for traffic on eastbound I-80 on June 27, 2006 [17]. In each of the three scenarios, all the vehicles in the simulation run an application that sends a dummy frame with the vehicle's location information every $300-400 \mathrm{~ms}$. These dummy frames can be replaced by any other application's frames that need to be disseminated to a distant area. As mentioned in Section III-B, beacon frames are not re-broadcasted, so only the application dummy frames will be re-broadcasted. To show the improvement provided by $p$-IVG, we ran experiments where the described application used either basic flooding, standard IVG, or $p$-IVG.

\section{B. Backoff Percentage}

To measure the impact of using $p$-IVG on reducing the channel contention, we calculate the backoff percentage. In 802.11, when a node is ready to send a frame, it first listens on the channel. If the channel is busy, the node backs off and tries to send later. So the backoff percentage is the ratio between the total number of backoffs to the total number of sending attempts, averaged over all of the vehicles. As channel contention increases, the backoff percentage increases. Table II summaries the backoff percentage for flooding, IVG, and $p$ IVG at low, medium, and high densities. Using $p$-IVG results in a dramatically lower backoff percentage than either flooding or IVG. This means that channel contention when using $p$-IVG is much lower than either of the other cases.

\section{Reception Rate}

To assess the impact of wireless collisions, we measured the average reception rate over time. If a frame was transmitted and no other vehicle received it, then the frame was considered to not be received. Either the frame experienced a collision, or no other vehicle was within $300 \mathrm{~m}$ of the sender. Figure 3 shows the reception rate for $p$-IVG, IVG, and flooding with low,

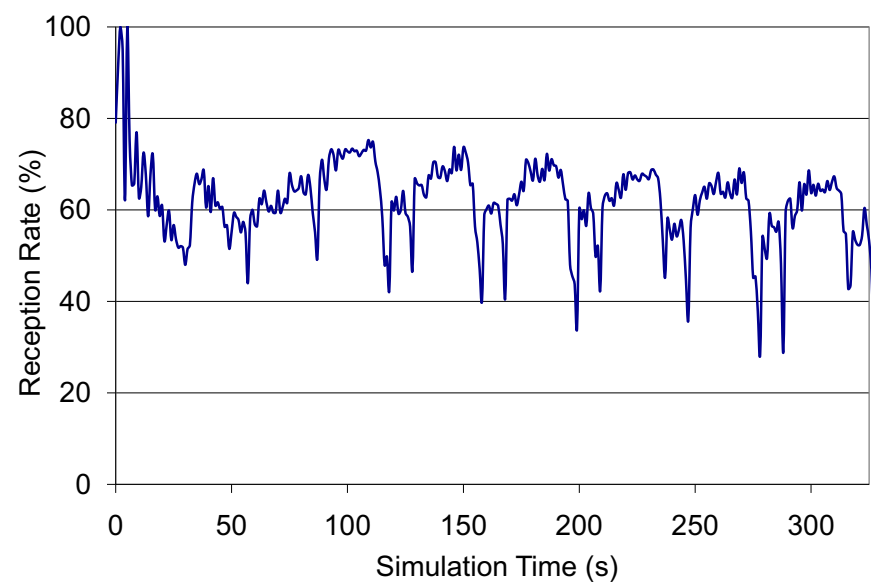

Fig. 4. Reception Rate with IVG at Medium Density

medium and high traffic densities, averaged every 10 seconds. The reception rates for IVG and flooding start high because the number of vehicles in the simulation is still increasing. As more vehicles enter the system, the reception rate decreases. Using $p$-IVG results in a very high reception rate for the entire simulation.

In our definition of reception, if just one vehicle receives the frame, it is considered to have been received. For $p$-IVG, high density traffic provides the best reception rate because there are more vehicles in range, thus there is a higher chance for a vehicle to receive the frame. The decreasing reception rate with low density is an artifact of how vehicles enter the simulation. Vehicles enter the highway with a low speed and gradually increase towards the maximum of $30 \mathrm{~m} / \mathrm{s}$. Until the last vehicle reaches the maximum speed, vehicles in front of it will be traveling faster, thus moving out of its transmission range. For low density, it takes longer for 500 vehicles to enter the system than with high density, thus it takes longer (in simulation time) for the last vehicle to reach $30 \mathrm{~ms}$.

To further demonstrate the problems we found with standard IVG, we show in Figure 4 the reception rate per second when using IVG at medium traffic density. Each drop spike in the reception rate corresponds to a situation where multiple vehicles at the boundary re-broadcast frames at the same time, resulting in the spatial broadcast storm problem.

\section{Dissemination Delay and Hops Count}

The main goal of any dissemination technique is carry the data as far as possible in the shortest amount of time, using the minimum number of hops. To assess the impact of using $p$-IVG on achieving this goal, in Figure 5 we show the average time a frame takes to travel a certain distance (dissemination delay) and the number of hops it visits along this trip (hops count) for $p$-IVG and IVG. $p$-IVG takes much less time and fewer hops to disseminate the data than IVG, while flooding failed to disseminate the data beyond $1 \mathrm{~km}$ and so was not pictured on the graph. With $p$-IVG, data was able to be disseminated up to $5 \mathrm{~km}$ in less than $500 \mathrm{~ms}$. 


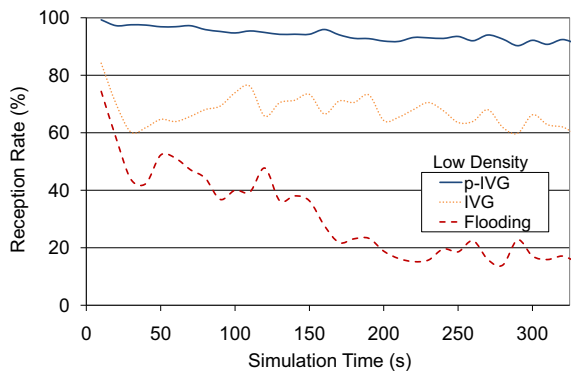

(a) Low Density

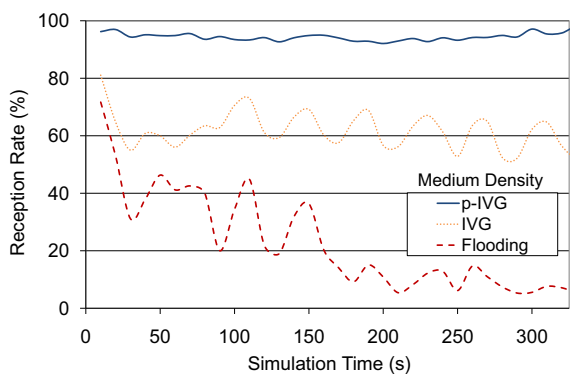

(b) Medium Density

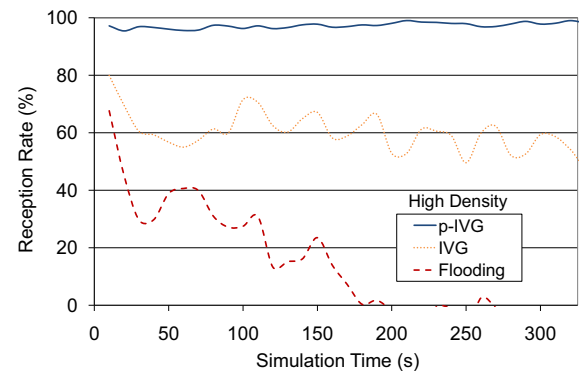

(c) High Density

Fig. 3. Reception Rate

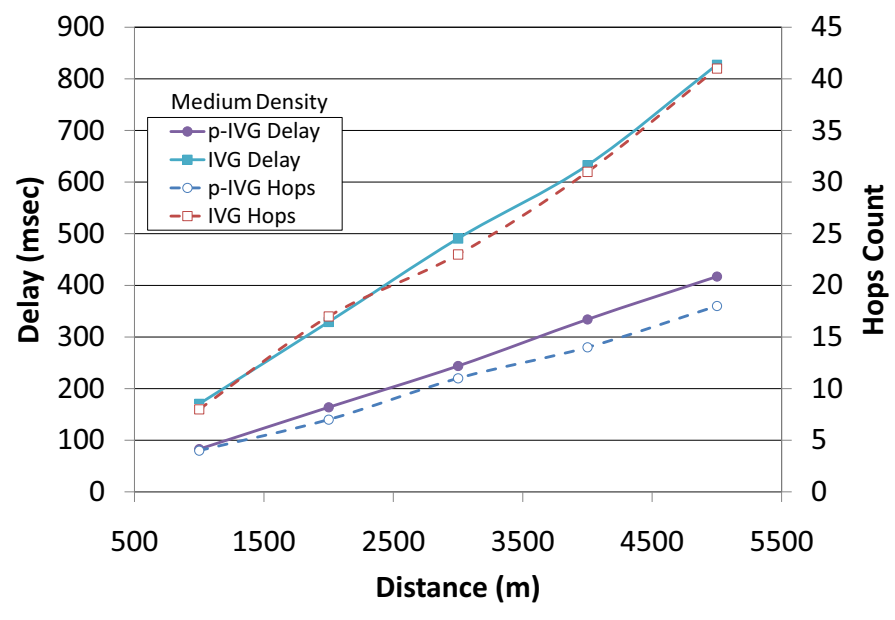

Fig. 5. Dissemination Delay and Hops Count at Medium Density

\section{CONCLUSION}

In this paper, we presented the probabilistic Inter-Vehicular Geocast ( $p$-IVG) protocol for data dissemination in dense VANETs. $p$-IVG was designed to alleviate the spatial broadcast storm problem that we discovered when studying standard IVG. $p$-IVG uses a light-weight beacon broadcasting utility to sense the local network topology and adapts itself to minimize the number of frame re-broadcasts needed to send a frame to distant area. We have shown that $p$-IVG has close to $100 \%$ reception rates, regardless of the traffic density, which is a large improvement over basic flooding and IVG. In addition, $p$-IVG reduces the channel contention, so that vehicles with data to send have a low probability of finding the channel busy. Finally, we have shown that $p$-IVG achieves the goal of disseminating data to distant areas in a short amount of time.

For future work, we will study the relationship between the $\varepsilon$ parameter and application data characteristics (sending frequency, length, etc.) in order to select the optimal value for each application.

\section{ACKNOWLEDGMENTS}

This work was supported by the National Science Foundation under Grant CNS-0721586.

\section{REFERENCES}

[1] S.-Y. Ni, Y.-C. Tseng, Y.-S. Chen, and J.-P. Sheu, "The broadcast storm problem in a mobile ad hoc network," in Proceedings of ACM Mobicom, Seattle, WA, Aug. 1999, pp. 151-162.

[2] N. Wisitpongphan, O. Tonguz, J. Parikh, P. Mudalige, F. Bai, and V. Sadekar, "Broadcast storm mitigation techniques in vehicular ad hoc networks," IEEE Wireless Communications, vol. 14, no. 6, pp. 84-94, Dec. 2007.

[3] M. Torrent-Moreno, D. Jiang, and H. Hartenstein, "Broadcast reception rates and effects of priority access in 802.11-based vehicular ad-hoc networks," in Proceedings of ACM VANET, Philadelphia, PA, Oct. 2004, pp. 10-18.

[4] A. Bachir and A. Benslimane, "A multicast protocol in ad hoc networks: Inter-vehicle geocast," in Proceedings of IEEE VTC-Spring, Jeju Island, Korea, Apr. 2003, pp. 2456-2460.

[5] O. Tonguz, N. Wisitpongphan, F. Bai, P. Mudalige, and V. Sadekar, "Broadcasting in VANET," in Proceedings of IEEE MOVE, Anchorage, AK, May 2007, pp. 7-12.

[6] Y.-C. Tseng, S.-Y. Ni, and E.-Y. Shih, "Adaptive approaches to relieving broadcast storms in a wireless multihop mobile ad hoc network," IEEE Transactions on Computers, vol. 52, pp. 545-557, 2003.

[7] C. Hu, Y. Hong, and J. Hou, "On mitigating the broadcast storm problem with directional antennas," in Proceedings of IEEE ICC, Anchorage, AK, May 2003, pp. 104-110.

[8] L. Briesemeister and G. Hommel, "Role-based multicast in highly mobile but sparsely connected ad hoc networks," in Proceedings of ACM Mobihoc, Boston, MA, Aug. 2000, pp. 45-50.

[9] K. Ibrahim and M. C. Weigle, "ASH: Application-aware SWANS with highway mobility," in Proceedings of IEEE MOVE, Phoenix, AZ, Apr. 2008.

[10] “JiST/SWANS," http://jist.ece.cornell.edu, 2004.

[11] R. Barr, Z. Haas, and R. van Renesse, Handbook on Theoretical and Algorithmic Aspects of Sensor, Ad hoc Wireless, and Peer-to-Peer Networks. CRC Press, 2005, ch. 19: Scalable Wireless Ad Hoc Network Simulation, pp. 297-311.

[12] F. Kargl and E. Schoch, "Simulation of MANETs: A qualitative comparison between JiST/SWANS and ns-2," in Proceedings of ACM MobiEval, San Juan, Puerto Rico, Jun. 2007, pp. 41-46.

[13] M. Treiber, A. Hennecke, and D. Helbing, "Congested traffic states in empirical observations and microscopic simulations," Physical Review E, vol. 62, no. 2, pp. 1805-1824, 2000.

[14] A. Kesting, M. Treiber, and D. Helbing, "MOBIL: General lane-changing model for car-following models," in Proceedings of the Transportation Research Board Annual Meeting, Washington, DC, Jan. 2007.

[15] J. Yin, T. ElBatt, G. Yeung, B. Ryu, S. Habermas, H. Krishnan, and T. Talty, "Performance evaluation of safety applications over DSRC vehicular ad hoc networks," in Proceedings of ACM VANET, Philadelphia, PA, Oct. 2004, pp. 1-9.

[16] N. Wisitpongphan, F. Bai, P. Mudalige, V. Sadekar, and O. Tonguz, "Routing in sparse vehicular ad hoc wireless networks," IEEE Journal on Selected Areas in Communications, vol. 25, no. 8, pp. 1538-1556, Oct. 2007.

[17] "Berkeley highway lab (BHL)," http://bhl.calccit.org/, 2006. 